\title{
Applying ABET Standards to Academic Engineering Programs (Field Study at the Technical Engineering College- Baghdad)
}

\author{
Hussien M. A. AL-mashhadany \\ Medical Devices - Institute of Medical Technology \\ Middle Technical University - Iraq \\ hmnmh7@yahoo.com
}

\author{
Murooj Naser Majeed \\ Electrical Techniques - Institute of Technology \\ Middle Technical University - Iraq \\ hmnmh7@yahoo.com
}

Received 17/9/2019

Accepted 18/12/2019

\begin{abstract}
:
The research aims to identify the standards of the American Engineering and Technology Accreditation Authority - ABET specialized in accrediting academic programs, including technical engineering, and see the view of teachers and lecturers at the Technical Engineering College - Baghdad, which is one of the formations of the Middle Technical University on the rate of achieving the criteria of students' output outcomes axis among graduates, Where these outputs represent one of the important axes of the requirements of the academic accreditation of the Engineering Accreditation Authority ABET, and to reach the opinions of teachers and lecturers at the college about the goal of the research a questionnaire has been designed that includes the axis of the educational outputs, which are 11 standards (a-k). the paper is based on Likert scale, the five-rank for the answers of the sample members, and it consists of (I agree totally and it has five degrees, I agree and it has four degrees, neutral and it has three degrees, I oppose and it has two degrees, very opposed and it has a score of zero) and the research sample consisted of the teachers of the Technical Engineering College - Baghdad and the lecturers from the Institute of Technology - Baghdad, a total of 63, and after analyzing the responses of the sample members about their expectations of the specifications of educational outputs according to the standards from (a-k). The research came out with a number of conclusions about the results of the questionnaire from general conclusions and some recommendations.
\end{abstract}

Keywords: ABET Standards, Academic Programs, Quality, Quality Assurance. 


\section{تطبيق معايير هيئة اعتماد الهندسة والتكنولوجيا الأمريكية ABET على البرامج الهندسية ( دراسة استطلاعية في الكلية التقنية الهندية-بغداد )}

\author{
مروج ناصر مجيد \\ التقنيات الكهربائية - معهد التكنولوجيا \\ الجامعة التقنية الوسطى - العراق \\ hmnmh7@yahoo.com
}

\author{
حسين محمد أحمد المشهاني \\ الأجهزه الطبية - المعهد الطبي التقني \\ الجامعة التقنية الوسطى - العراق المعدئ \\ hmnmh7@yahoo.com
}

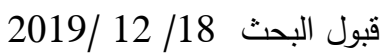

استلام البحث 17 / 2019/

الملخص:

يهدف البحث إلى التعرف على معايير هيئة اعتماد الهندسة والتكنولوجيا الأمريكية - ABET التتخصصة في اعتماد البرامج الأكاديمية

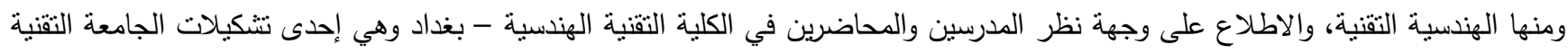
الوسطى، وعلى نسبة تحقيق معايير محور المخرجات التعليمية Students Outcomes لدى الخريجين، حيث تمثل تلاك المخرجات أحد المحاور المهمة لمنطلبات الاعتماد الأكاديمي لهيئة اعتماد الهندسة والتكنولوجيا ABET، وللوصول إلى آراء المدرسين والمحاضرين في الكلية حول هدف التهات

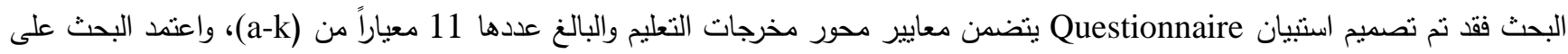

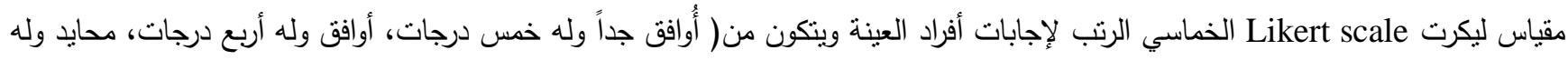

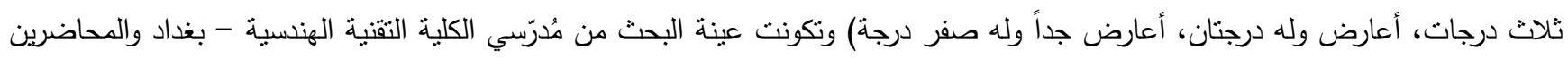
من معهد التكنولوجيا-بغداد والبالغ عددهم 63 عضو هيئة تدريس، وبعد تحليل إجابات أفراد العينة حول توقعاتهم لمواصفات المخرجات التعليمية

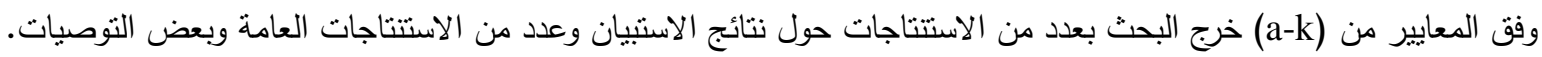
الكلمات المفتاحية: (معايير الجودة).ABET ، البرامج الأكاديمية، الجودة، ضمان.

المقدمة:

السعي والإعداد للحصول على الاعتماد الأكاديمي الدولي أو المحلي

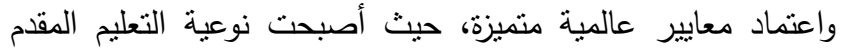

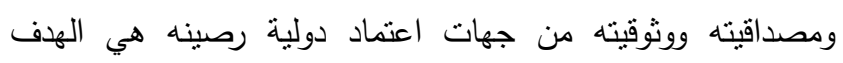

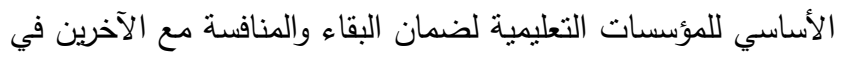

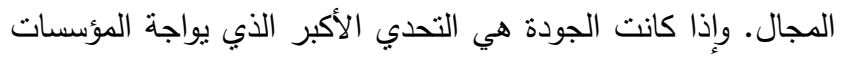

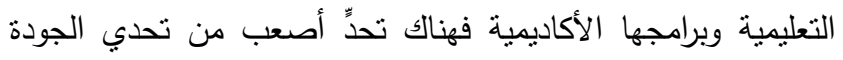

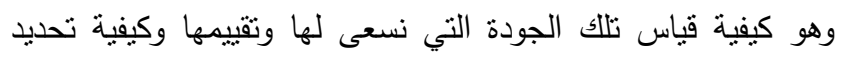
مصادر جمع البيانات والأدلة التي تعكس مدى تحقيق النتائج والتأكد من بلوغ الجودة في التعليم التي نصبو اليها ودرجة اقترابنا أو ابتعادنا منها، حيث نتبلور على أساس ذلك نوعية الفعاليات التعليمية

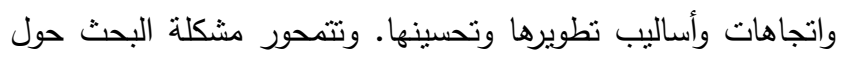
كيفية قياس تلك الجودة وضمان نوعية المخرجات التعليمية الدقصودة من نلك البرامج.
شهد التعليم العالي في المنطقة العربية ومنها العراق توسعاً ملحوظاً من حيث أعداد الجامعات الحكومية والأهلية وأعداد الملتحقين

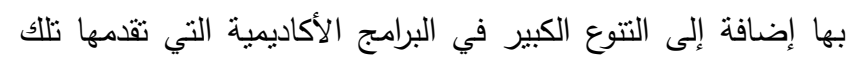

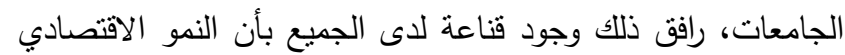
والاجتماعي لا يمكن أن يحدث دون برامج تعليمية متميزه ورصينه، وتعززت تلك القناعة من خلال وجود الفجوة الكبيره بين مخرجات

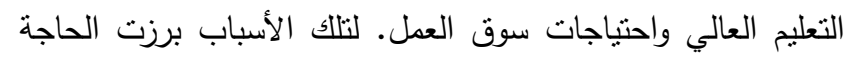

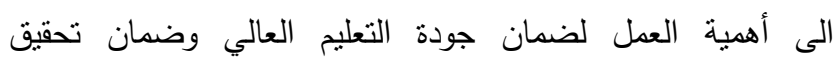
المخرجات التعليمية المقصوده والتي تتتاسب مع احتباجات الجهات fitness for purpose المستقيده من الخريجين لكل تخصص وللوقوف على فجوات الأداء ورصد أسبابها وتحليل بيانات التغذية الراجعة من متابعة الخريجين وملاحظات الجهات المستفيدة

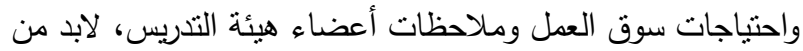


بشكل طوعي للخضوع للاراسة الثاملة والفحص والاختبار وكانت قد قامت بتتفيذ أهدافها التعليمية المحددة لها بشكل فعال وقد هيأت البنى التحنية والمستلزمات التي تُكن الطالب من تحقيق الأهداف المخططه

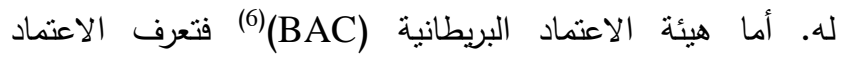
الأكاديمي على أنه منزلة Status أو شهاده تمنح إلى البئه البرنامج الأكاديمي الذي يؤمّن معايير محدده لوجود النعليم المتميز، معتمدين في ذللك على منظومة مؤسسية للتقييم لها معاييرها الخاصة عند منح

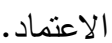

\section{البرنامج الأكاديمي Academic program:}

يُعرف من قبل(ABET)(7)، على أنهائه تجربة منظمه منكامله

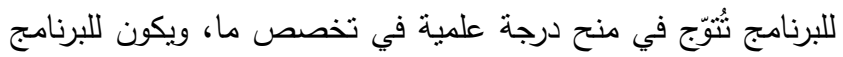

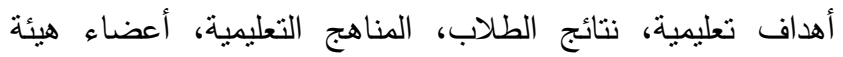
التدريس، الإمكانيات. أما (الحولي)(8)، فقد عرف البطابل البرنامج الأكاديمي

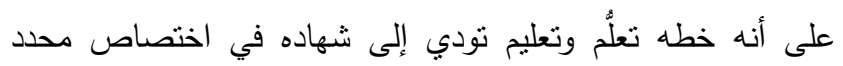

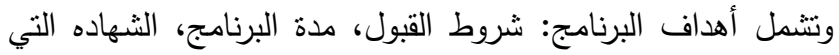
يؤدي إليها، نوع الثهاده أكاديمية أم مهنية.

\section{جودة التعليم Quality Education:}

مجموعة من المعايير والمقاييس المتفق عليها من قبل هيئات وطنية أو دولية تمنح الاعتماد الأكاديمي، ويؤشر تتفيذ تلك المعايير والمقاييس إلى بيئة نعليمية متميزه في تحقيق أهدافها التعليمية المقصودة والمحدده لها، وتوفر بيئة التعليم الجيد المعرفة والمهارات

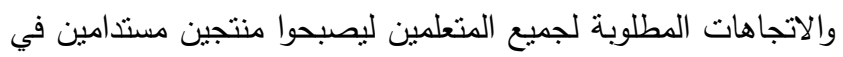

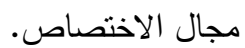

\section{ضمان الجودة Quality Assurance:}

وتعرف بأنها جميع السياسات والإجراءات والتدابير والعطليات المخططة للحفاظ على جودة التعليم وتحسين معاييره .وتتصف عمليات ضمان الجودة بالاستمرارية والمنهجية في تقييم جميع الأنشطة

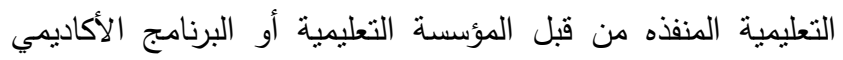
وتمثل عمليات ضمان الجودة جزءًا من إدارة جودة التعليم.

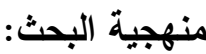

اعتمد البحث منهج دراسة الحاله case study لملاحظة فعل قد انقضى وهو تخريج دفعات من البرنامج التقني الهندسي من الكلية

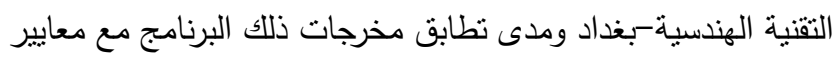

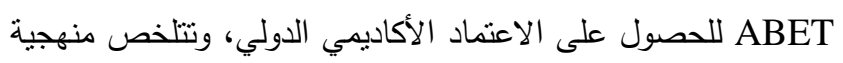

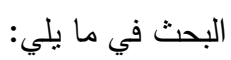
1. دراسة الأدبيات المتاحه حول معايير ABET لكنح الاعتماد الأكاديمي للبرامج الهندسية التقنية.

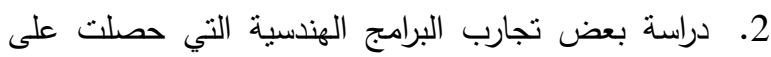
الاعنماد الدولي لهيئة ABET.

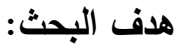

يهدف البحث إلى تحقيق مايأني:

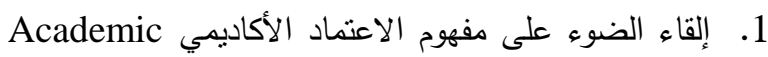

.Accreditation

2. التعرف على معايير الاعتماد الأكاديمي للبرامج الهنسية

التقنية كما حددنها ABET.

3. تحليل آراء الددرسين في الكلية التقنية الهندسية حول تحقيق

معايير مخرجات التعلم Students Outcomes ضمن البرنامج الهندسي الذي يقدمونه للطلبة.

وتفيد نتائج البحث المؤسسات الراغبة في الحصول على الاعتماد الاكاديمي الدولي ABET بالإضافه إلى:

1. الجهات المستفيده من مخرجات البرامج الهندية المعتمده.

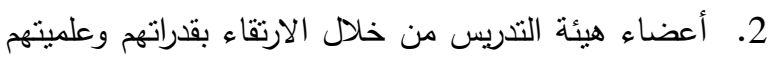
من أجل تحقيق معايير الاعتماد. 3. الطلبة الملتحقين بالبرامج المعتمدة وعوائلهم لاطمئنانهم على الاعلى

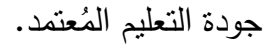
وكانت حدود البحث ضمن الكلية التقنية الهندسيه - بغداد للعام الدراسي 2018-2019.

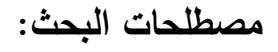

\section{المعيار standard}

تُعرفه الهيئة الأوربية للمقاييس (ECS)(1)،بأنه عباره عن وثيقه

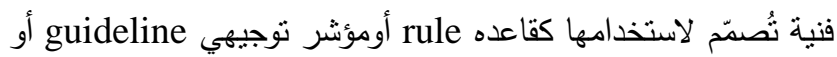
تعريف definition. وتُعرفه الأكاديمية العربية البريطانية للتعليم العالي (ABAHE) (2)،على أنه وثيقه تحدد المتطلبات التي يجب أن النية

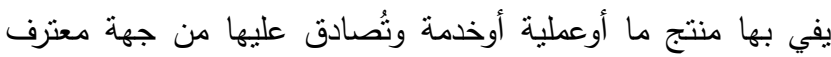
بها. أما Oxford dictionary فيعرف المعيار على أنه مستوى مطلوب أو متفق عليه من الجودة أو التحصيل. المعايير الأكاديمية Academic criteria: يعرفها مكتب ضمان الجودة البريطاني (QAA)(3) على أنها لإنها مستوى الإنجاز الذي يتعين على الطالب بلوغه للحصول على شهادة التهاني

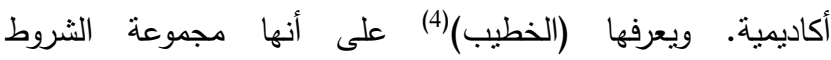

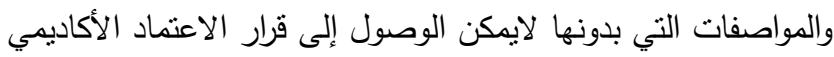

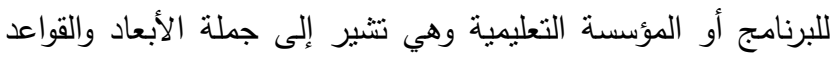

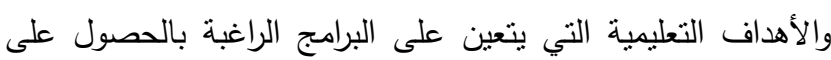

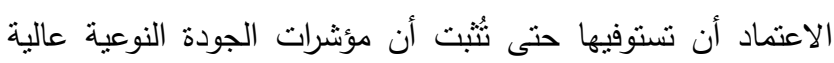

\section{الاعتمادالأكاديمي Accreditation:}

يُعرفه (Mohieldin) (5) على أنه شهادة Certificate تُمنح من قِبل هيئة متخصصة معترف بها، بأن المؤسسة التعليمية قد تقدمت 
مساعدة الطلبة وأولياء أمورهم على الالتحاق بالبرامج

العلمية المُعتمدة للتاكد من جودة العملية التعليمية.

توضح للجامعات والكليات أفضل الآليات التي يمكن

اتباعها لقياس وتقييم تحسين جودة التعليم في برامجها

$$
\text { التعليمية. }
$$

ولغاية 2019 فقد منحت ABET الاعتماد الأكاديمي إلى 4005 برامج أكاديمية في 793 كلية وجامعة موزعة على 32 بلدأ ويتخرج أكثر من برامج 85000 طالبٍ سنويا من برامج ABET المُعتمدة وهناك

الماليين ممن تخرجوا تحت تلك المعايير (www.ABET.org). وتتخصصABET بمنح الاعتماد الأكاديمي للبرامج الهندسية (EAC)، البرامج التكنولوجية (TAC)، برامج العلوم التطبيقية وبرامج علوم الكومبيونز (CACAC) (ASC) ولاتقوم بمنح الاعتماد الأكاديمي للأشخاص أو الأقسام العلمية أو الكليات أو الدرجات العلمية أو المؤسسات التعليمية وهي ليست نظاماً لتصنيف Ranking إنما هي شكل من أشكال ضمان الجودة للبرامج الأكاديمية واعتمادها، وهو معترف به عالميا كدليل على أن البرنامج المعتمد يفي بالمعايير

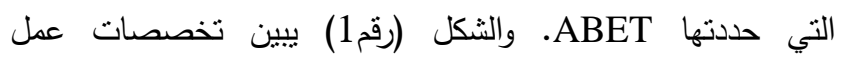
ABET والبرامج التي تمنح الاعتماد لها.

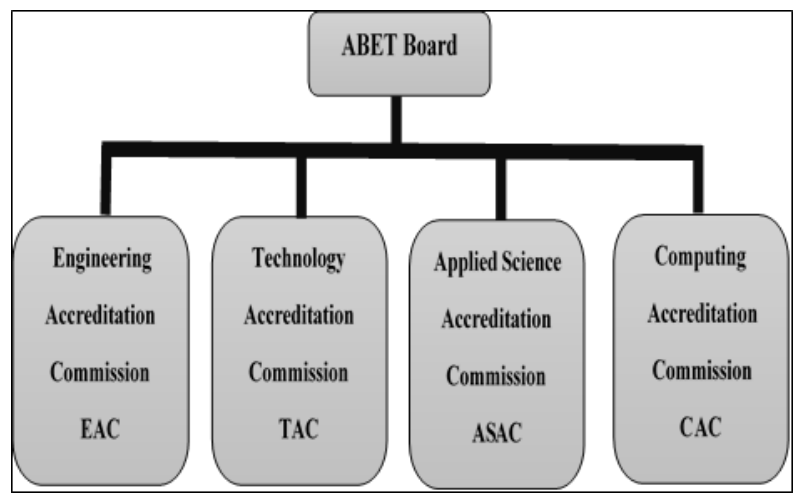

شكل (رقم 1): تخصصات عمل ABET.

ويتمثل دور ABET في تقديم تقييم خارجي External Assessment الأكاديمي وبشكل دوري ومستمر ودعم برنامج تحسين الجودة. ويتم اختيار المقيّمين الخارجيين من المختصين في تخصص البرنامج من جهات فنية متخصصة وسوق العمل والأقران في المجال من خلال فريق عمل، وتقدم ABET التقييمات والحلول حول الممارسة المهنية

$$
\text { والإعداد المهني والتحسين المستمر للجودة. }
$$

\section{خطوات منح اعتماد ABET:}

1. يتقدم البرنامج الأكاديمي بطلب رسمي طوعي للمراجعة والاعتماد على أن يكون له دفعة من الخريجين على الأقل.
3. التعمق في دراسة معايير ABET للبرامج الهندسية التقنية Engendering Technology محاور من خلا الأدلة الإرشادية وإصدارات ABET. 4. بناء استبيان يوزع على أعضاء هيئة التدريس والمحاضرين ضمن البرنامج التعليمي للكلية يتضمن معايير المحور الثالث أونتائج التعلم Students Outcomes المتوقعة لاى الخريجين كما حدنتها ABET لمنح الاعتماد الأكاديمي للبرامج الهندسية. 5. ثم جاءت الخطوة الأخيره في تحليل نتائج الإجابات وتصنيفها بجداول إحصائية ومناقنتة نتائجها والخروج بعدد من الاستتناجات والتوصيات التي تتعلق بموضوع البحث.

$$
\text { الجانب النظري: }
$$

$$
\text { معايير الاعتماد الأكاديمي الدولي: }
$$

إن المتتبع لعملية بناء المعايير الدولية يجد أنّ معظم المعايير قد نم بناؤها وفق فلسفة الدوله المعنية وتوجهاتها العلمية وأهدافها التربوية، وتم إعدادها من قبل خبراء ومهنيين ووكالات ومؤسسات متخصصة مستقلة، ثم تم تجريب تللك المعايير على عينة من الجامعات والتخصصات لإثبات صلاحيتها. وقد كانت ثللك الجامعات سباقة في تطبيق هذه المعايير والحصول على شهادة الاعتماد الدولي من أجل الدخول في المنافسة مع الجامعات الاخرى واستقطاب

$$
\text { الطلاب للالتحاق بها (سوسن) (9). }
$$

ولعل أشهر وكالات منح الاعتماد الأكاديمي للبرامج الهندسية التقنية في الولايات المتحدة الامريكية هي هيئة اعتماد الهندسة (Accreditation Board for Engineering والتكنولوجيا ومقرها ولاية ميرلاند، وهي منظمه غير Technology-ABET) ربحية أنشئت عام 1932م لمنح الاعتماد الأكاديمي وضمان الجودة

$$
\text { لبرامج التعليم العالي. }
$$

وتتعاون معها 30 جمعية علمية ومهنية وهندسية ويشارك فيها أكثر من 1500 من ذوي الخبره الأكاديمية والصناعية في إجراءات

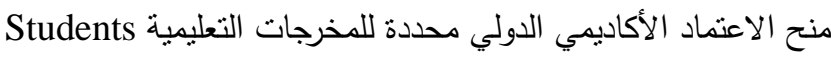
يutcomes

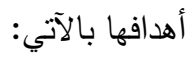

التأكد من استعداد خربجي البرنامج المُعتمد على الاخول والاستمرار في ممارسة مهنة الهندسة. زيادة تنافسية الخريجين في سوق العمل. منح الطلاب إمكانية الانتقال إلى جامعات مناظرة. ضمان اعتراف جامعات العالم والمؤسسات التعليمية الأخرى بخريجي البرنامج المُعتمد ومعادلة شهادتهم وقبولهم في برامج الدراسات العليا. 
• تُعبر الأهداف النعليمية عن محتويات المنهج النظرية والعطلية وتتتاسب مع تخصص البرنامج. • • توصيف إجراءات ومسارات لتحقيق الأهداف التعليمية

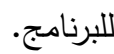
• وجود آليات وتعليمات وقوانين مرجعية لضمان قياس التقدم الأكاديمي للطالب.

• وجود أهداف تعليمية واضحة ومُحدة وموثقة لكل مقرر . 3. مخرجات التعليم Students Outcomes: كل برنامج هندسي تقني يجب أن يبين بأن مخرجاته تمتلك المزايا

$$
\text { الآتية من (a-k) وهي: }
$$

an ability to القدرة على an القيق المعرفة في مجال a

$$
\text { الرياضيات والعلوم والهندسة. }
$$

b. القدرة على تصميم واجراء التجارب وتحليل البيانات. c. القدرة على تصميم نظام أو عملية لتلبية الاحتياجات ضمن ولفيل

\section{تخصصه.}

ed القدرة على العمل في فرق متعددة الاختصاصات. e. القدرة على تحديد وحل المشكلات الهندسية في مجال فلى فئل

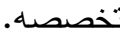

f القدرة على فهم المسؤلية الأخلاقية والمهنية للمهنة. g g. القدرة على التواصل مع الآخرين بفعالية وكفاءه. h. القدرة على التعليم الواسع لفهم تاثير الحلول الهندسية في سياق عالمي واقتصادي وبيئي واجتماعي والرغبة في ذلك. i أقدرة على التعليم مدى الحياة والاستشعار بأهمية ذلك. j. القدرة على معرفة القضايا المعاصرة. k القدرة على استخدام التقنيات والمهارات والأجهزه والمعدات

$$
\text { الهندسية اللازمه لممارسة المهنة. }
$$

وتصف مخرجات البرنامج بما يمكن للطلاب إظهاره من حيث المعرفه والمهارات والقيم عند الانتهاء من البرنامج وتمنل النتائج أعلاه

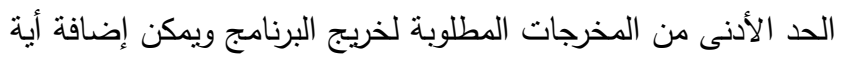
معارف ومهارات يراها البرنامج ضرورية التكوين لاى الخريجين.

4. التحسين المستمر Continuous Improvement:

يستخدم البرنامج وبانتظام عمليات نقييم مناسبة وموتقة،

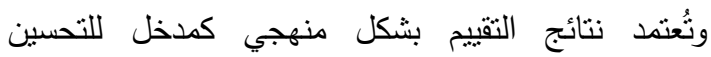
المستمر للبرنامج.

5. المناهج Curriculum: يجب أن يتصف المنهج

الدراسي للبرنامج بما يأتي: • يحد متطلبات التخصص. • ب بضضمن وقتاً كافياً بما يحقق أهداف البرنامج.
2. تقدم ABET عرضاً تفصيلياً لمنطلبات الاعتماد ويتطلب

ذللك امتتال البرنامج للمعايير والسياسات والإجراءات التي تقوم بها ABET. 3. أن يكون اسم البرنامج ضمن توصيفات البرامج التي تقوم

ABET

4. تعرف ABET البرنامج على أنه تجربه متكاملة ومنظمة وتتوج بدرجة علمية وله أهداف نعليمية محددة ومنهج تعليمي وأعضاء هيئة تدريس. 5. يقدم البرنامج دراسة ذاتية Self-study Report تقصيلية عن مجمل نثاطاته ويهيئ الوثائق التي تؤيد الإنجاز للتأكد من جاهزية البرنامج التعليمي لمعايير ABET.

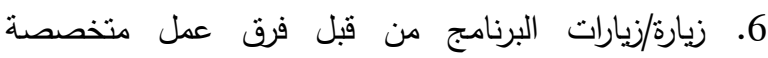
بموضوع البرنامج. 7. في نهاية عمليات الفحص والتدقيق والمناقثة مع الإدارة والمدرسين والطلاب يقدم فريق العمل تقرير زيارة Visit Report يتضمن بيانات نقاط القوه في البرنامج وأوجه القصور ونقاط الضعف والهخاوف Concerns وذلك استتاداً إلى الوثائق والملاحظات خلال الزياره الموقعيه. 8. نستمر الزيارات واللقاءات بالعاملين في البرنامج والطلبة

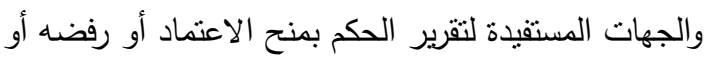
تأجيله، وتكون القرارات سرية.

معايير الاعتماد الأكاديمي لهيئة ABET: حدد ABET (9) المعايير الأكاديمية لبرنامج الهندسة

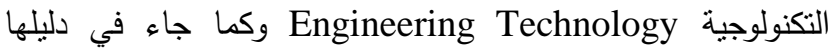
الارشادي 2019-2020 على النحو التالي: 1. الطلاب Students: يجب أن يؤكد البرنامج على قياس أداء الطلبة، مراقبة تقدمه الأكاديمي، بناء عناصر المهنة لديهم، يعزز نجاحات الطلبة باتجاة نحقيق الأهداف التعليمية الدقصوده، توفر الرغبة في تخصص البرنامج، شروط قبول الطلبة عادلة وفعالة ومتجاوبة مع احتباجاتهم. 2. PEOProgram الأهداف التعليمية للبرنامج Educational Objective والحصول على الاعتماد لا بُدّ من أن تلبّي الأهداف التعليمية مجموعة من المتطلبات منها: • تتضمن الأهداف التعليمية رؤية البرنامج لتكوين المعرفة والههارات الفنية والهندسية القابلة للقياس. • نشر الأهداف التعليمية للطلبة والددرسين. • ضمان إنرالك الجهات المستقيدة من مخرجات البرنامج بمراجعة الأهداف التعليمية. 
• وجود بيئة جامعية جاذبة لاستقطاب الكفاءات من أعضاء هيئة التدريس.

• الهيئة التدريسية لها خلفية تعليمية وقاعدة تكنولوجية ومعلومات فنية تدعم التخصص.

\section{T الإمكانيات Facilities:}

وتتضمن إمكانيات البرنامج الأكاديمي البنى التحتية من قاعات

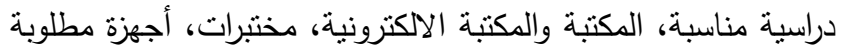

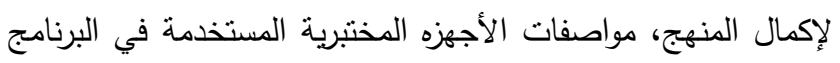
متطورة عما هو موجود في الصناعة والحياة العملية. البنى التحتية من الحواسيب وتكنولوجيا المعلومات والانترنيت وأجهزة الحاسوب المتقدمة مع برامجها المستخدمة تناظر المستخدم في الحياة العملية والجهات المستفيدة من مخرجات البرنامج.

8. الدم المؤسسي Institutional Support: ويتضمن الدعم المؤسسي ما يأني:

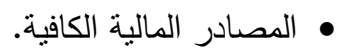

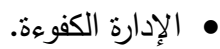

• • الإرشاد الأكاديمي للبرنامج Advisement.

وتقع معايير ABET كما جاء في دليلها الإرشادي 2012-

2013 في قسمين هما:

a يجب على كل البرامج باختلاف تخصصاتها أن تستوفيها

\section{وكما حدنتها ABET.}

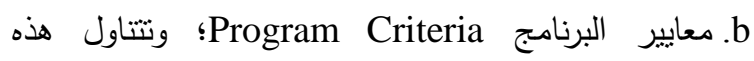
المعايير المنطلبات الخاصة بتخصص البرنامج والتي تفي بشهادة وعنوان البرنامج ويتم نطوير هذه المعايير من قبل

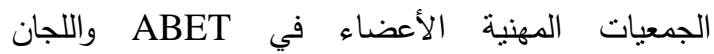
التخصصية في مجال البرنامج، ويجب نطبيق معايير كلا

$$
\text { القسمين مرةً واحدة. }
$$

\section{مفهوم مخرجات التعل Students Outcomes:}

تمثل عملية تحويل معايير الاعتماد الأكاديمي الدحلي أو

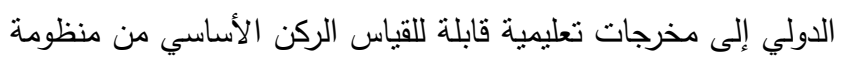

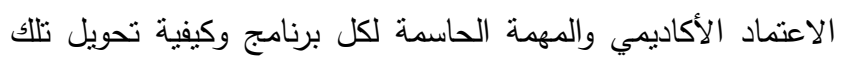
المعايير إلى أداءات وأفعال واتجاهات وقيم تتعلق بالجانب المعرفي

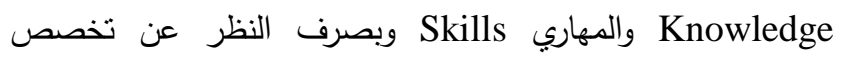
البرنامج. وتعتبر إجراءات قياس الجودة ومعاييرها عملية معقدة وكثيرة الحوار والجدل وتتطلب قيادات ماهرة من جانب القائمين عليها، كما تتطلب الوقت الكافي للإنجاز ومحاكاة المستقبل ومحاورة جميع الأطراف المستقيدة من مخرجات البرنامج واستيعاب كافة طروحاتهم
• تتوفر خطة تفصيلية لتنفيذ كل مقرر دراسي وتتضمن الوصف العام للمادة والهدف منها. • يتضمن أساليب التدريس والتدريب والتقويم وتوزيع الدرجات. • • ل بتوفر الكتاب المقرر والمراجع الساندة. • يتوافق المنهج الدراسي مع الأهداف التعليمية للبرنامج.

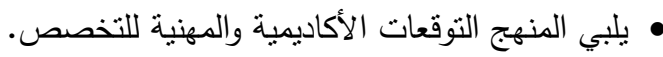

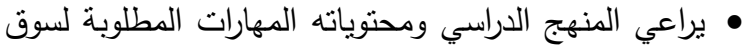
العمل والجهات المستقيدة.

• براعي المنهج النطورات والاتجاهات الحديثة المرتبطة بمجال التخصص.

ويتميز البرنامج الهندسي التقني كما تراه ABET a يزود المحتوى التقني الأساسي Technical Core a بقاعدة عريضة من المعلومات التقنية التخصصية ويتدرج بالتقنيات المعقدة.

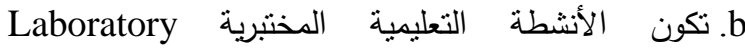
Activities استخدام وقراءة وتحليل مخرجات الأجهزه والمعدات وأجهزة القياس في مجال تخصصده وضمن أهداف البرنامج. c تتصف المقررات الثقنية Technical Courses بقدرتها على تتمية معرفة الطالب وتطوير قدراته في استخدام معايير التصميم والتتغيل والصيانه وآلياته العملية فيلية الصحيحة بما يتلاءم مع الأهداف التعليمية للبرنامج.

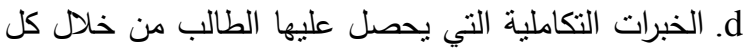
المقررات الأكاديمية وغير الأكاديمية تتمي قدرته في حل المشكلات التقنية.

Communication تتمية قدرات الطالب في الاتصال e ويتضمن التخطيط والتنظيم والإعداد وتحرير التقارير الثقتية الفعالة المكتوبة أو الثفهيه. 6. أعضاء هيئة التدريس Faculty: • وجود مدرسين متفرغين بدوام كامل من حيث العدد والخبرة وتخصصات متتوعه تلائم تخصص البرنامج. • كافة المجالات المعرفية لتخصص البرنامج مغطاة من قبل المدرسين ونسبة من المحاضرين. • وجود آليات مناسبة للتعيين والتزقية والتقييم لأعضاء هيئة التدريس وبشكل دوري. • دعم مشاركة أعضاء هيئة التدريس في المؤتمرات والنشاطات العلمية. • تتوفر الحرية الأكاديمية لأعضاء هيئة التكريس لتقويم وتطوير المناهج. 
• نطوير الأنشطة التعليمية الني من شأنها أن تؤثرّ على مخرجات الطلبة متل، المناهج الدراسية، سياسات القبول، البنى التحتية، دعم النواصل مع الخريجين وسوق العمل والجهات الستفيدة من خريجي البرنامج.

وترى ABET أن الاعتماد الأكاديمي للإرج الهندسية يحقق

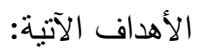

كاف التأكد من أن خريجي البرنامج الأكاديمي مستعدون وبشكل كاف للاخول والاستمرار في مهنة الهندسة. • تحفيز جودة التعليم وتحسين التعليم الهندسي.

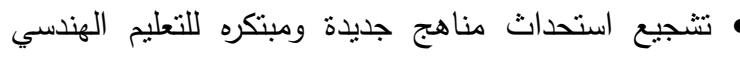
واختبارها.

\section{الاراسات السابقة Literature Review: (12)(Robinson) دراسة}

تهدف إلى تحديد الخصائص والمهارات والمعرفة والمواقف والقيم لكل معيار من معايير ABET ضمن برنامج الهندسة

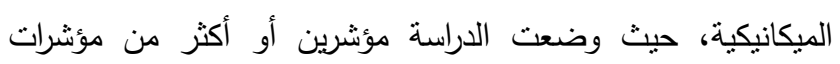

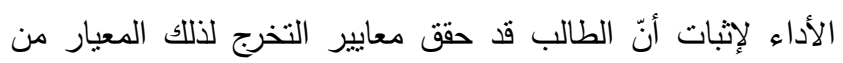

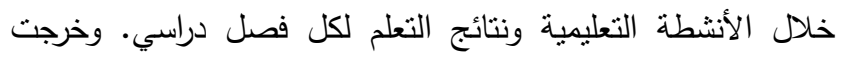
الدراسة بتحويل جميع معايير الاعتماد الأكاديمي إلى قائمةٍ من الإنا مؤشرات أداء قابلة للقياس Performance indicators-PI واضحة ساعدت الددرس على متابعة تحقيق الأهداف التعليمية وضمان العمل على نحقيق مخرجات التعلم Learning Outcomes، ولم تضف الدراسة أية معايير محلية إضافية. دراسة (عماد الدين) (13)

تناول البحث دراسة تجربة كليات الهندسة في جامعة الخرطوم

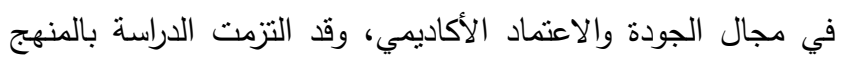
الوصفي التحليلي في معالجتها لموضوع الدراسة حيث نم تقييم تجربة كليات الهنسة في الحصول على اعتماد ABET ومن ثم معرفة المعوقات والتحديات التي واجهت التجربة، وخرجت الدراسة بعدد من

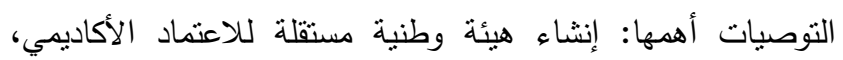

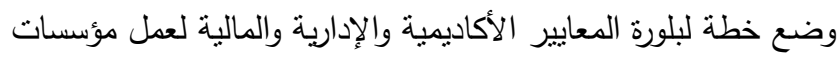

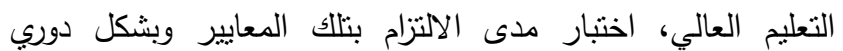
ومستمر ، وتزويد الهيئة بالتغذية الراجعة للاستفادة منها للتطوير لئري والتحسين في جميع المجالات.

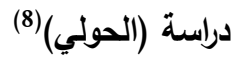

وتضمنت اقتراح آليات لعملية تطوير البرامج الأكاديمية في مؤسسات التعليم العالي مستخدماً المنهج الوصفي التحليلي مستفيداً

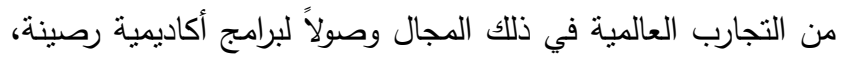

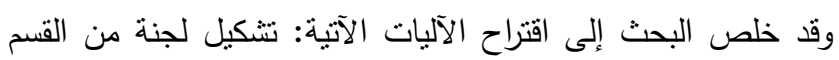

وملاحظاتهم حول إعداد الطالب وبما يضمن تقليص الفجوة بين الإعداد واحتياجات سوق العمل، ثم تاتي بعدها قدرة المناهج التعليمية

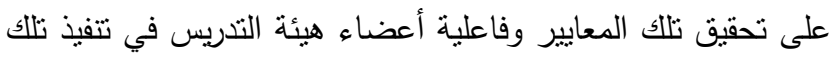

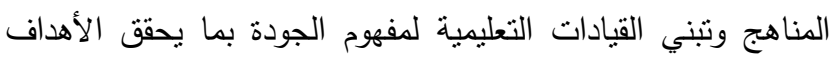

التعليمية المقصودة.

وتمثل مخرجات التعلم أحد المحاور الأساسية للاعتماد الأكاديمي لهيئة ABET حيث يتكون من أحد عشر معياراً من (a-k) وتركز تلاك المعايير كما في البرنامج الهندسي التقني على نطبيقات المعرفة في مجال الرياضيات والعلوم والهندسة وتصميم الأنظمة وبناء التجارب المختبرية وقراءة بياناتها وتحليلها إضافة إلى أخلاقيات المهنة

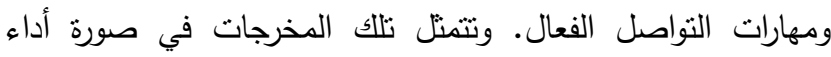
Performance المخرجات ما يتم إنجازه من قبل الطلبة بعد تخرجهم وتقييم تلك ألكان الإنجازات ضمن بيئة العمل الحقيقية للخريج. وتشتهدف مخرجات الطلاب أنواع المعرفه الآتية (Biggs)(10): 1. معرفة المحتوى Context Knowledge: وهي معرفة مقررات البرنامج.

2. المعرفة الإجرائية Procedure Knowledge: وهي معرفة كيفية القيام بالأشياء.

3. المعرفة الثرطية Condition Knowledge: وهي معرفة منى تُفعل الاشياء. 4. المعرفة التوظيفية Function Knowledge: معرفة كيفية توظيف الأنواع الثلاثة من المعارف لحل المشاكل والعمل

$$
\text { كمتحرف ومتميز · ت كوصن }
$$

وتتثبر (سوسن) (11) إلى أنّ الاهتمام بنواتج الطلبة يعني مايأني:

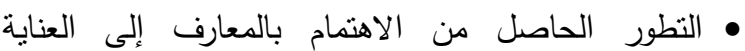

$$
\text { باستخدام المعرفة. }
$$

• التحول في التركيز من مدخلات العملية التعليمية إلى بلى الترفة مخرجات العملية التعليمية ونواتج الطلبة.

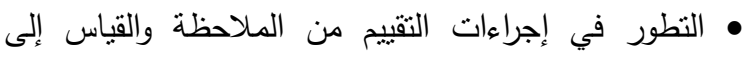
· العمل بالمعايير

$$
\text { وتأني أهمية محور مخرجات الطلبة من الآتي: }
$$

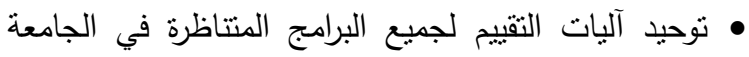
الواحدة. • تفعيل عمليات المراجعة المستمره لمخرجات الطلاب وتقييمها والتحسين المستمر لها. • التركيز على مختلف الجوانب المعرفية والتطبيقية وتطوير القدرات والمهارات الفنية والهندسية للطالب بما يحقق نتائج التعلم المحددة للبرنامج الأكاديمي. 
Statistical Techniques العمليات الإحصائية

نم استخدام العمليات الإحصائيه الآتية (فهمي)(15):

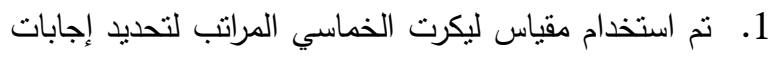

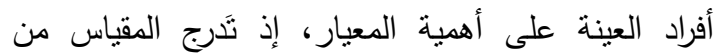

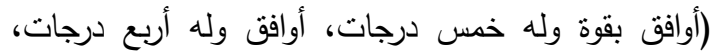

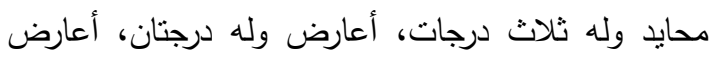
بقوة وله صفر درجة).

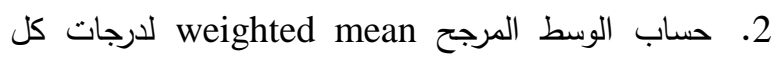
معيار حصل عليها من إجابات أفراد العينة والنتيجة كلما اقترب الوسط المرجح من 5 ارتفعت درجة قبول المعيار

$$
\overline{x_{1}}=\frac{\sum S 1+S 2+S 3+S 4+S 5}{N}
$$

× عدد التكرارات للمعيار تحت مرتبة المقياس S (1-5)

$$
\text { درجة مرتبة المقياس. }
$$

3. الوزن المئوي للتكرارات لكل مرنبة من المقياس الخماسي

التي حصل عليها المعيار وحسب المعادلة الآتية:

$$
\text { \% } \frac{S(1-5)}{N}
$$

4. الوزن المئوي الكلي لقبول المعيار وحسب المعادلة الآتية:

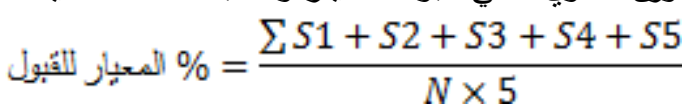

\section{تحليل الاستبيان Analysis of Questionnaire:}

اعتمد البحث مقياس ليكرت Likert scale الخماسي ويعتمد

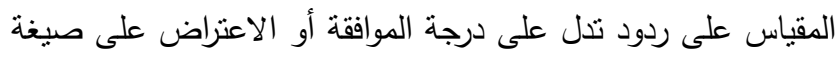
ما لتقدير درجة توقع أفراد العينة من أعضاء هيئة التدريس لنتائج التعلم students outcomes من (a-k) كما حددتها ABET. وتم تحديد درجة القبول استتاداً إلى النسبة المئوية الكلية لإجابات أفراد

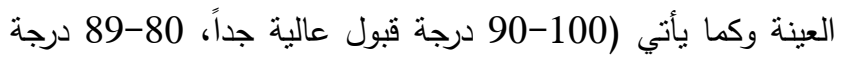
قبول عالية، 70-79 درجة قبول متوسطة، 60-69 درجة قبول ضعيفة، 59 فما دون درجة قبول مرفوضة)، والجدول رقم (1) يبين

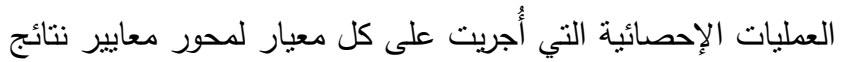

$$
\text { الطلبة (ملحق رقم 2). }
$$

1. بينت نتائج إجابات أفراد العينة عدم وجود درجة قبول بمستوى (عالية جداً) لأيٍّ من معايير مخرجات الطلبة، وظهرت درجة قبول بمسنوى (عالية) لأربعة معايير ، ودرجة

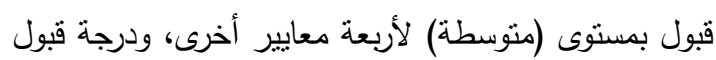

العلمي المعني للقيام بمهمة تطوير البرنامج الأكاديمي، استطلاع آراء

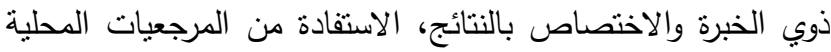
والعربية والدولية المتخصصة في هذا المجال، اثنمال البرامج

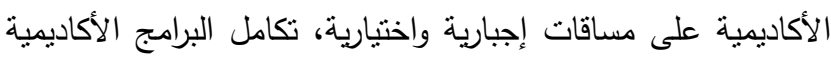
مع مخرجات المراحل الثانوية، مراعاة النطور فيها، عقد ورشة عمل الثلانه لمناقثة البرنامج الأكاديمي المقترح بمشاركة أعضاء هيئة الندريس

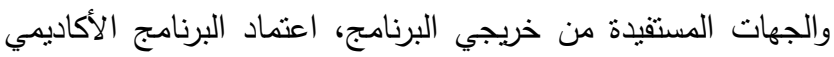
المقترح من قبل القسم العلمي من خلال محضر رسمي بذلك.

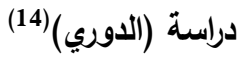

هدفت الدراسة إلى قياس التوافق بين الدخرجات الفعلية للبرامج

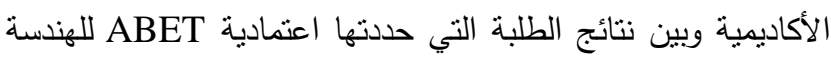

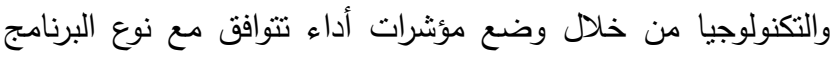

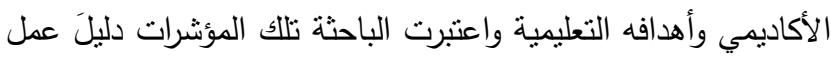
للبرامج التي تسعى للحصول على اعتماد ABET وذللك لتقليص الفجوة بين الأداء الفعلي والأداء المرغوب به من جهة، ولضمان تحقيق منطلبات الجهات المانحه للاعتماد من جهة ثانية وبالتالي ضمان الحصول على متطلبات الجهات المانحة للاعتماد. واختارت

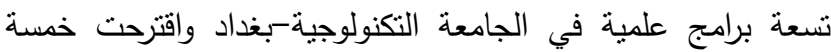
مؤشرات لقياس المخرجات التعليمية وهي (الطلبة والقدرات المعرفية والإبداعية، المهارات المتعددة لأعضاء الهيئة التنريسية، المناهج

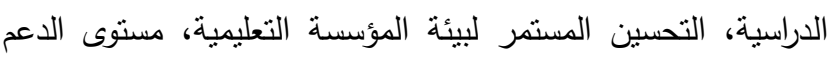
والإداره والإثراف من الإدارة العليا) ولتحقيق هدف البحث فقد صُمدت لئن

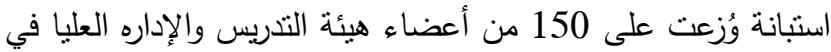
البرامج وبعض الطلبة لاختيار النوافق بين مخرجات البرنامج العلمي ونتائج الطلبة كما في ABET.

\section{Practical Framework الإطار التطبيقي}

أداة الدراسة Study Instrument

اقتصرت أدوات جمع المعلومات على الاستيان الموزع على عينة البحث إضافةً إلى الملاحظة الثخصية observation للباحثين كونهما من أعضاء هيئة التريس، حيث تم إعداد استنبان مغلق يتكون من معايير محور مخرجات التعلم Students Outcomes كما نراها ABET لاعتماد البرامج الهندسية، وقد تكون الاسنتيان من

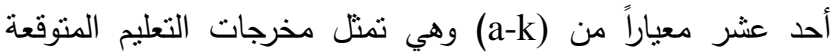
والمرغوبة والمخطط لها للطلبة ومدى قدرتهم على ممارستها والعمل بها بعد التخرج. وتم استخدام مقياس ليكرت الخماسي لتنرج إجابة ولية العينة على المعايير (ملحق رقم 1).

\section{Study Population مجتمع الدراسة}

يتكون مجتمع الدراسة من المدرسين والمحاضرين في برنامج الكلية. 
المعيار (d) والمعيار (g) وبنسبة 29\% لكل منهما واللذان يثيران إلى (القدره على العمل في فرق متعدارة الاختصاصات والقدرة على التواصل مع الآخرين بفعالية

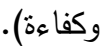

6. احتلت المعايير الثلاثة الآتية أقل نسب لإجابات (أوافق

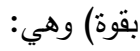
المعيار (j) والمعيار (h) وبنسبة 13\% واهي: الكل منهما واللذان يشيران إلى (القدره على معرفة القضايا المعاصره والقدرة على التعليم الواسع والرغبة في ذللك). المعيار (e) وبنسبة 16\% والذي يشير إلى (القدرة على الى تحديد وحل المشكلات الهنسية في مجال تخصصد).

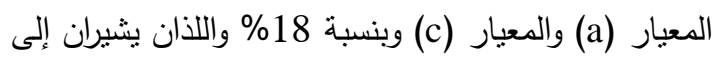
(القدره على تطبيق المعرفة في الرياضيات والعلوم والهنسة (c) ولفئة والقدرة على تصميم نظام أو عملية لتلبية الاحتياجات المطلوبة ضمن تخصصد). 7. الاستتاجات أعلاه تمثل وجهة النظر لعينة من أعضاء هيئة التدريس في مكان وزمان محددين وتعكس حالة

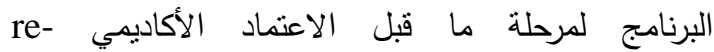
accreditation بلوغ الاعتماد الأكاديمي الدولي لابد من الاعتراف بوجود المشكلة والسعي الدائم لتجاوز معيقات تنفيذ المعايير الدولية لاعتمادية برامجها، ولا تكتمل الصورة عند تلك الملاحظات بل هي بحاجة شديدة إلى آراء الجهات المستقيدة من لن مخرجات البرنامج وهما سوق العمل والطلبة أنفسهم واعتماد ملاحظات التخذية العكسية كمنهاج عمل للنطوير.

\section{(المناقشة Conclusions:}

نتتوع أساليب وطرق قياس مخرجات التعليم وتختلف باختلاف طبيعة الدقرر الدراسي واختلاف أهدافه التعليمية، ومن أدوات القياس 1. المقابلات الثخصية Interviews مع الطلبة الخريجين، الفيات العاملين في البرنامج الأكاديمي، الجهات المستقيده، سوق العقات

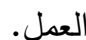

2. الامتحانات المعيارية الموحدة Standardized Exams. 3. الزيارات الميدانية لعمل الخريج.

4. الامتحانات التحريرية والثفهية الداخلية Locally الفصلية والنهائية للبرنامج . 5. مراجعة وتصحيح عمل الطلبة النظري أو العملي.

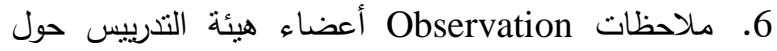
طريقة تتفيذ العمل من قبل الطالب وتقييم مؤشرات الأداء
بمستوى (ضعيفة) لثلاثة معايير، ولم نَظهر النتائج درجة قبول بمستوى (مرفوضة) لأيّ معيار . 2. حصلت ثلاثة معايير على أقل النسب المئوية من الإجابات وبمستوى قبول (ضعيفه) وهي: المعيار (e) وبنسبة موافقة كلية بلغت 60\% والذي يشير إلى (القدرة على تحديد وحلّ المشكلات الهندسية في مجال تخصصد). المعيار (b) وبنسبة موافقة كلية بلغت 63\% والذي يشير إلى (القدرة على تصميم وإجراء التجارب وتحليل وتفسير

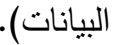
المعيار (c) وبنسبة قبول كلية بلغت 66\% والذي يشير إلى (القدرة على تصميم نظام أو عمليات لتلبية الاحتياجات المطلوبة للتخصص) 3. احتلت المعايير الثثاثة الآتية أعلى النسب المئوية للموافقة على توفرها لاى الطالب الخريج وتحت درجة قبول (عالية) وهي: المعيار (f) وبنسبة موافقة كلية بلغت 84\% والذي يشير إلى (القدرة على فهم المسؤلية المهنية والأخلاقية للمهنة). المعيار (k) وبنسبة موافقة كلية بلغت 82\% والذي يشير إلى (القدرة على استخدام التقنيات والمهارات والاجهزه والمعدات الحديثة لممارسة الهندسة). المعيار (a) وبنسبة موافقة كلية بلغت 80\% والذي يشير إلى (القدرة على an ability to تطبيق المعرفة في العلوم والهنسة والرياضيات). 4. بلغت نسبة إجابات أفراد العينة تحت تقدير (أوافق بقوة) ولجميع المعايير بنسبة 24\%، وظهرت نحت تقدير (أوافق) بنسبة 47\%، وظهرت تحت تقدير (محايد) بنسبة 26\%،

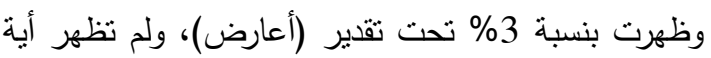

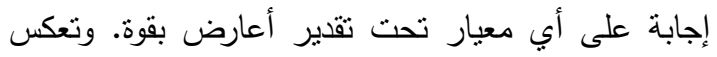

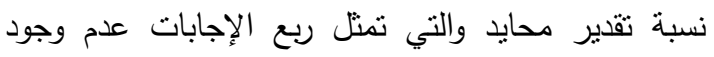
تصور واضح لاى عينة البحث حول مخرجات الطلاب الهتوقعة من البرنامج، حيث وصلت تللك النسبة إلى 50\% حول المعيار (h) وبنسبة 39\% حول المعيار (i). 5. صلت ثلاثة معايير على أعلى النسب وبمستوى إجابة (أوافق بقوة) وهي: المعيار (k) وبنسبة 42\% والذي يثبر إلى (القدره على استخدام التقنيات والمهارات والأجهزة والمعدات الهندسية الحديثة اللازمة لممارسة المهنة). المعيار (b) وبنسبة 31\% والذي يشير إلى (القدره على تصميم وإجراء التجارب وتحليل وتفسير البيانات). 


\section{الاستنتاجات Results:}

1. استطاعت الكلية التقنية الهندسية (مكان الدراسة) تحقيق معايير ABET للبرامج الهندسية التكنولوجية من وجهة نظر أعضاء هيئة التدريس بنسبة 71\% عند درجة قبول (أوافق بقوة) ودرجة قبول (أوافق)، وهي نسبة جيدة لتحقيق الاعتماد الأكاديمي مستقبلا وتمثل بداية مشجعه للشروع في الإعداد

$$
\text { للحصول على الاعتماد الأكاديمي الدولي. }
$$

2. البرنامج بحاجة ماسة إلى تحديد الفعاليات المطلوبة لمرحلة ماقبل الاعتماد re-Accreditation للوصول بنسبة لاتقل مالك عن 90\% عند درجة قبول (أوافق بقوه) لجميع محاور ABET قبل تقديم الطلب للحصول على الاعتماد الأكاديمي الدولي. 3. وجود مدى واسع بين إجابات أعضاء هيئة التدريس حول تحقيق محاور ABET حيث تراوحت نسبة إجابات أفراد العينة على مديات نطبيق محاور الاعتماد الأكاديمي عند درجة قبول (أوافق بشدة) بين نسبة مئوية تتراوح من 13\% إلى 42\%، وهي نسبة تعكس عدم وضوح الأهداف لتحقيق تلك المعايير حيث تؤكد ABET على تتفيذ جميع المعايير

$$
\text { المحددة دون استثناء. }
$$

4. افتقار الفعاليات التعليمية إلى سياقات ثابتة ومستمرة لتعميق دون قدره الطلبة على فهم القضايا المعاصرة التي تهم البلد والمنطقة والعالم وفهم تاثير الحلول الهندسية للمشاكل التتموية والصناعية والخدمية وفق منظور عالمي واقتصادي h, وبيئي واجتماعي وانعكس ذلك على درجة قبول المعيارين بنسبة لم تتجاوز 13\% 5. عدم وجود قنوات تحمل صفة الاسنمرارية والثبات لمتابعة الخريجين مما أدى إلى انعدام التغذية العكسية الراجعة من بيئة العمل من قبل الخريجين وأرباب العمل والجهات المستقيدة والتي تعتبرها ABET كمنهج للتطوير لجميع الأنشطة التعليمية للبرنامج وتطوير المناهج وسياسات القبول.

\section{التوصيات Recommendations:}

1. إنشاء هيئة وطنية لمراجعة الاعتماد الأكاديمي مستقلة إدارياً

$$
\text { ومالياً تقوم بالمهام الآتية: }
$$

دراسة الطلبات المقدمة من قبل المؤسسات التعليمية للحصول على الاعتماد الأكاديمي الدولي المؤسسي أو البرامجي أو المهني والتحقق من استيفائها شروط وأحكام الجهات المانحة للاعتماد.
(Performance Indicators -PI)

لكلأهداف التعليمية المحددة. 7. مراجعة الواجب المنزلي Home work والتقاربر والمشاريع

المقدمة. - مراجة

$$
\begin{aligned}
& \text { 8. فاعلية الطالب ودوره في العمل الجماعي العلمي } \\
& \text { أوالاجتماعي. }
\end{aligned}
$$

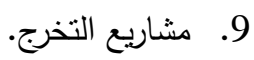

ومن المهم جداً اختيار الطريقة المناسبة لقياس كل هدف تعليمي، فمثلاً الامتحانات الكتابية غير مفيدة لقياس درجة اكتساب المهارات ولكنها مناسبة لقياس المعرفة العامة للبرنامج وبالتالي لايمكن أن يكون هناك سؤال واحد ونمط واحد من الأسئلة مناسب لقياس

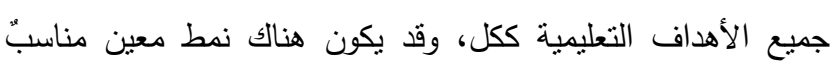
لقياس تحصيل بعض المعايير ولا يصلح لجميع تلك المعايير . وعند قياس مخرجات التعلم للمعيار (b) والمتضمن (القدرة على تصميم التجارب) فإن بعض مؤشرات الأداء PI الممكنة لاستخدامها في التقييم هي أن بقوم الطالب بما بأني: 1. بختار الطريقة المناسبة بين عدد من الأساليب التجربيية الممكنة في تصميم التجربة المطلوبة.

2. يختار تقنيات جمع البيانات المناسبة الممكنة Possible candidates 3. تصميم تجربة تتنج بيانات فعاله وحاسمة لتحديد النتائج المستهدفه. 4. يقدم تحليلاً دقيقاً لنقاط القوه والضعف للتجربة المصدمة

.Experimental design

Outside ومن الضروري استخدام المقيم الخارجي assessors

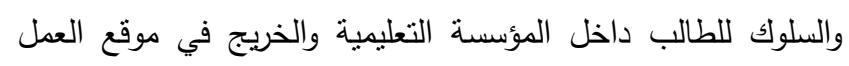
أثتاء قيامه بأداء المهمة ذات الصلة بالبرنامج وتقييم المعرفة والمهارة التي يُظهرها أثثاء إنجاز العمل، وهكا يتم تحويل جميع معايير مخرجات الطلبة من a-k إلى مؤشرات أداء تعكس درجة تحقيق تلك المخرجات المرغوب بها. ولضمان دقة التقييم يجب صباغة مؤشرات الأداء بأسلوب وعبارات واضحة وقابلة للقياس ومن خلال مقياس متعدد المستويات (تناتي أو رباعي أوخماسي) واستخدام أدوات تقييم متعددة للوصول إلى تقييم نتائج محددة، وبالتالي يجب أن يكون التقييم

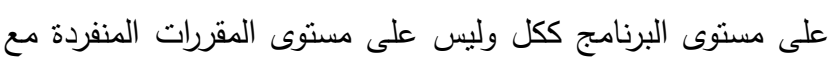
أهمية وجود حاجة إلى تقييم موثوق لكل مخرجات الطلبة المحددة للبرنامج. وقد اتفقت الدراسة مع أغلب الدراسات التي اطلع عليها الباحث على أهمية تحويل معايير الاعتماد إلى معايير فرعية متعددة واضحة وقابلة للقياس وتعكس حالة تحقيث الأهداف التعليمية وقياسها وتقييمها وتحسينها بشكل مستمر . 
تطبيق معايير هيئة اعتماد الهندسة والتكنولوجيا الأمريكية ABET على البرامج الهندسية ( دراسة استطلاعية في الكلية التقنية الهندسية - بغداد ).

5. Mohieldin, TAJ (2006) "Hand book of Assessment and Accreditation" University of Bahrain - college of Engineering, Kingdom of Bahrain.

6. WWW.the-bac.org, BAC-British Accreditation Council (2018), The last visit 7/10/2019, Accreditation handbook, London.

7. WWW.abet.org / The Accreditation Board for Engineering and Technology-ABET, The last visit 15/11/2019 Accreditation policy and procedure manual, October 27/2012, USA.

8. Al-Houli, Ehlian Abdullah (2014) "Proposed mechanisms for the development of academic programs in institutions of higher education-Palestine" Arab International Conference IV to ensure the quality of higher education Zarqa University, Jordan.

9. - WWW.abet.org / The Accreditation Board for Engineering and Technology, The last visit 15/11/2019. Criteria for accreditation Engineering Technology program, manual (2019-2020), USA.

10. Biggs (1999)"Formulating and clarifying curriculum objectives "ch: 3 , Society for Research Higher Education and Open University - U.K.

11. Shaker, Sawsan Majid (2011) "Academic Standards: General Outlook" The Second International Symposium on the Outstanding Performance of Organizations and Government, University of Ouargla, Algeria.

12. Robinson, Risa and Edward, Hensel (2015) "Engineering education Facing the grand challenges, what we doing" $13^{\text {th }}$, LACCEI Annual international conference july29-31, Santo Domingo, Dominican Republic.

13. Emad EI-Dean، Hassan Ahmed (2013)، Quality standards for academic programs, faculties of engineering, University of Khartoum, Model of the Third Arab International Conference for Quality Assurance of Higher Education, Zaytoonah University, Jordan.

14. Al-dorrie, Maha Abdel-Karim Hammond (2017) "Concordance between the outputs of students according to the reliability of ABET and the indicators of measuring outputs of academic programs" The ninth

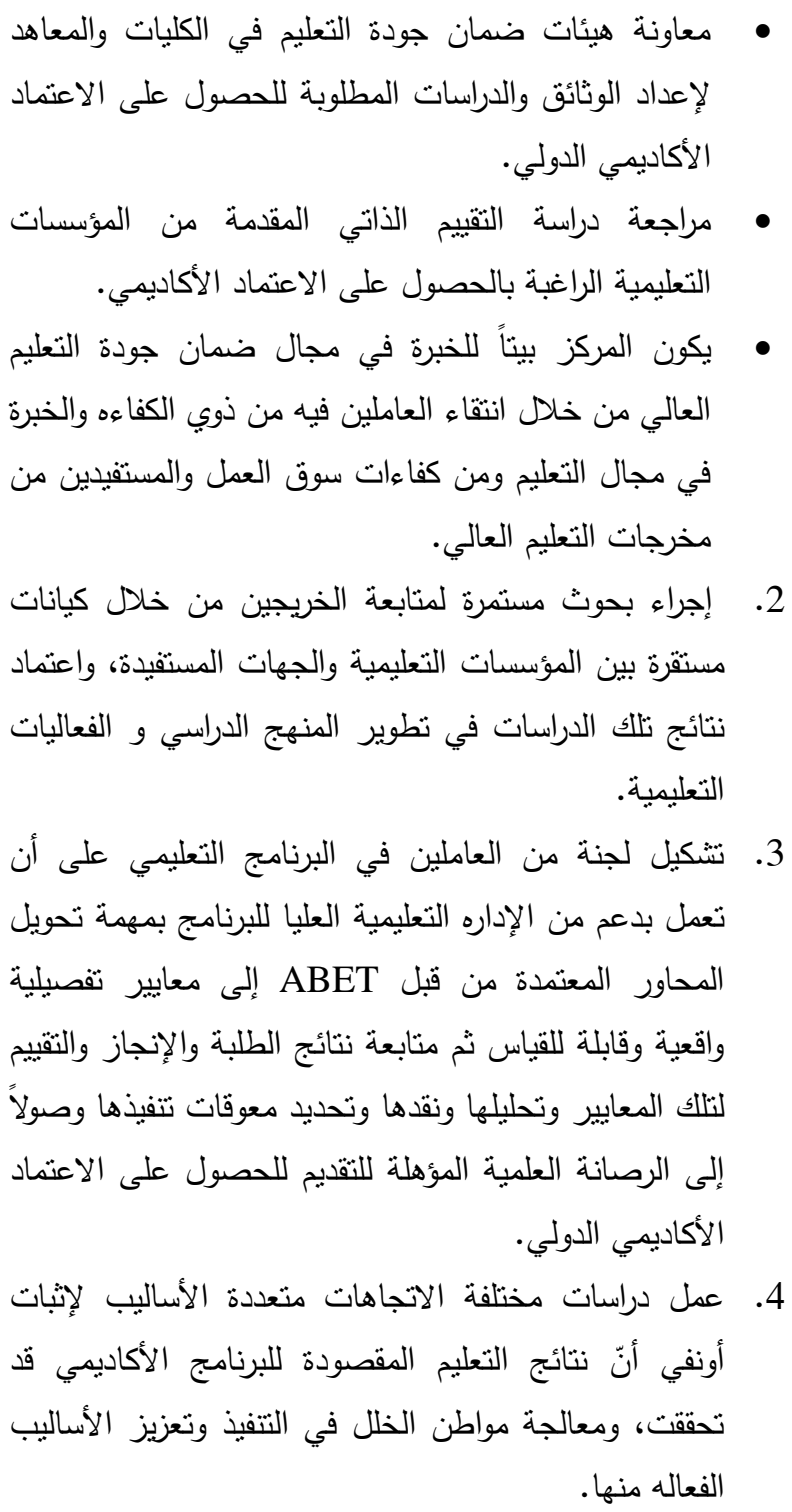

\section{References:}

1. WWW.CEN.EU/ European Committee for Standardization-U.K, The last visit 12/10/2019.

2. WWW.ABAHE_UK/ Arab British Academic for Higher Education, The last visit 11/15/2019.

3. WWW.qaa.ac.uk / The Quality Assurance Agency for H.E, The last Visit 11/14/2019 "ENQA Agency Review: QAA for higher education", U.K.

4. Al-Khatib, Mohamed (2002) "Standards of Accreditation and Quality Assurance " a study presented to the meeting of experts on quality assessment and accreditation of higher education institutions in the Arab Gulf States in cooperation with UNESCO, Ministry of Higher Education, Sultanate of Oman. 
annual conference of the Arab Organization for Quality Assurance in Education - challenges and prospects "Arab League - Cairo.
15. Fahmy, Abdel Aziz (1996), "Principles of Statistical Methods", Dar al-Mahdi alArabiya, Beirut.

\section{ملحق رقم 1 مكان}

السادة أعضاء هيئة التدريس المحترمون: يهدف البحث إلى دراسة إمكانية تطبيق معايير الهيئة الامريكية لاعتماد البرامج الهندسية ABET والتي تتكون من تسعة محاور أحدهما (نتائج الطلبة outcomes students).... يرجى وضع إثناره على مستوى القدرات الذي تراه عند الطالب الخريج. ونؤكد بأن إجاباتكم وملاحظاتكم لأغراض البحث فقط وهي موضع تقدير واهتمام. اللقب العلمي .

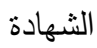

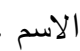

\begin{tabular}{|c|c|c|c|c|c|c|}
\hline أعارض بقوة & 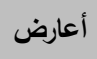 & 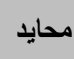 & أوافق & أوافق بقوة & المعيار & $ت$ \\
\hline & & & & & القدرة على An ability to تطبيق المعرفة في الرياضيات والعلوم & $\mathrm{a}$ \\
\hline & & & & & القدرة على تصميم وإجراء التجارب وتحليل وتفسير البيانات . & $\mathrm{b}$ \\
\hline & & & & & القدرة على تصميم نظام system أوعمليةsprocess لنليبة الاحتياجات & $\mathrm{c}$ \\
\hline & & & & & القدرة على العمل في فرق متعددة الاختصاصات. & $\mathrm{d}$ \\
\hline & & & & & القدرة على تحديد وحل الششكلات الهنسية في مجال تخصصه. & $\mathrm{e}$ \\
\hline & & & & & القدرة على فهم المسئولية المهنية والأخلاقية للمهنة & $\mathrm{f}$ \\
\hline & & & & & القدرة على التواصل مع الآخرين بفعالية وكفاءة (ثفهي وتحريري). & $\mathrm{g}$ \\
\hline & & & & & القدرة على التعليم الواسع لفهم تاثير الحلول الهندسية في سياق عالمي & $\mathrm{h}$ \\
\hline & & & & & القدرة على التعليم مدى الحياة واستشعار أهمية ذلك . & $\mathrm{i}$ \\
\hline & & & & & القدرة على معرفة القضايا الدعاصرة. & $\mathrm{j}$ \\
\hline & & & & & القدرة على استخدام التقتيات والمهارات والأجهزة والمعدات الهندسية & $\mathrm{k}$ \\
\hline
\end{tabular}




\section{ملحق رقم 2}

\begin{tabular}{|c|c|c|c|c|c|c|c|c|c|}
\hline درجة القبول & الوزن المئوي & الوسط المرجح & أعارض بقوة\% & 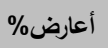 & 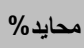 & أوافقى\% & أوافق بقوة \% & المعيار & \\
\hline عالية & 80 & 4 & - & - & 19 & 63 & 18 & القدرةعلى تطبيق المعرفه في الرياضيات والعلوم & $\mathrm{a}$ \\
\hline ضعيفة & 63 & 3.15 & - & 9 & 18 & 42 & 31 & القدرة على تصميم وإجراء التجارب وتحليل وتفسير & $\mathrm{b}$ \\
\hline 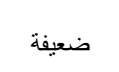 & 66 & 3.3 & 3 & 12 & 31 & 39 & 18 & القدرة على تصميم نظام أو عملية لتلبية الاحتياجات & $\mathrm{c}$ \\
\hline منوسطة & 74 & 3.7 & - & 3 & 26 & 42 & 29 & القدرة على العمل في فرق متعددة الاختصاصات. & $\mathrm{d}$ \\
\hline 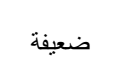 & 61 & 3.04 & - & 1 & 31 & 52 & 16 & القدرة على تحديد وحل الششكلات الهنسية في مجال & e \\
\hline عالية & 84 & 4.18 & - & - & 8 & 66 & 26 & القدرة على فهم المسئولية المهنية والأخلاقية للمهنة & $\mathrm{f}$ \\
\hline عالية & 82 & 4.1 & - & - & 18 & 53 & 29 & القرة على التواصل مع الآخرين بفعالية وكفاءة & $\mathrm{g}$ \\
\hline متوسطة & 72 & 3.63 & - & - & 50 & 37 & 13 & 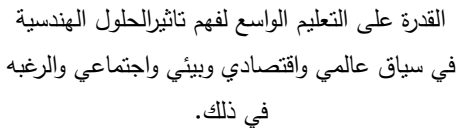 & $\mathrm{h}$ \\
\hline متوسطة & 76 & 3.76 & - & 3 & 39 & 37 & 21 & القدرة على التعليم مدى الحياة واستشعار اهمية ذلك. & $\mathrm{i}$ \\
\hline منوسطة & 76 & 3.81 & - & 1 & 31 & 55 & 13 & القدرة على معرفة القضايا المعاصرة. & $\mathrm{j}$ \\
\hline عالية & 82 & 4.08 & - & 9 & 18 & 31 & 42 & والمعدات الهنىسية الحديثة اللازمة لممارسة المهنة المتنة. & $\mathrm{k}$ \\
\hline
\end{tabular}

